\title{
Percutaneous Cryoablation of Multiple Pulmonary Endometriosis
}

\author{
Chong Hoon Kim, M.D. ', Doo Yun Lee, M.D. ', Duk Hwan Moon, M.D.', Sungsoo Lee M.D., Ph.D. ${ }^{1}$ \\ 'Department of Thoracic and Cardiovascular Surgery, Gangnam Severance Hospital, Yonsei University College of Medicine, Seoul; ${ }^{2}$ Department of Thoracic and \\ Cardiovascular Surgery, G SAM Hospital, Gunpo, Korea
}

\section{ARTICLE INFO}

Received February 27, 2020

Revised June 28, 2020

Accepted July 3, 2020

Corresponding author

Doo Yun Lee

Tel $82-2-2019-3380$

Fax 82-2-3461-8282

E-mail CHESTLEE@yuhs.ac;

dylee4831@chamc.co.kr

ORCID

https://orcid.org/0000-0003-3817-7066

\begin{abstract}
Minimally invasive cryoablation is often considered for lung tumor patients with high surgical risk or inoperable metastatic lung tumors. Cryoablation is a type of thermal percutaneous ablation in which argon and helium gases are delivered via a cryoprobe to induce tissue freezing and necrosis. We report the case of a 23-year-old woman who had suffered from multiple pulmonary endometriosis with frequent intermittent hemoptysis during menstruation for 6 years prior to her visit. She was treated with cryoablation at our hospital, and since her treatment, she has been doing well with no hemoptysis for at least 6 months. Although endometriosis is a benign lung disease, cryoablation is an ideal and effective treatment option for patients with multiple endometriosis.
\end{abstract}

Keywords: Cryosurgery, Endometriosis, Argon and helium gases

\section{Case report}

We report the case of a 23-year-old woman who had suffered from intermittent hemoptysis during menstruation for 6 years prior to seeking medical care. After receiving an initial diagnosis of pulmonary endometriosis at a local hospital, the patient reported at our institution wanting a complete cure of hemoptysis with eradication of the pulmonary endometriosis using a minimally invasive surgical method. A gynecological examination showed no evidence of pelvic endometriosis.

A full check-up, including chest computed tomography (CT), was performed at the Department of Thoracic and Cardiovascular Surgery of G SAM Hospital. Chest CT showed multiple endometrioses in both lungs (Figs. 1, 2A); hence, complete eradication of endometriosis was not possible, even with multiple wedge resections (Fig. 1). As a result, cryoablation was considered and performed as an initial treatment to destroy the 2 large endometrial lesions in the right lung.

Cryoablation with real-time CT was performed in a radiology intervention room at G SAM Hospital on January 23,2019 . The cryoablation system involved argon and helium gases used with disposable cryoprobes with 2 sharp

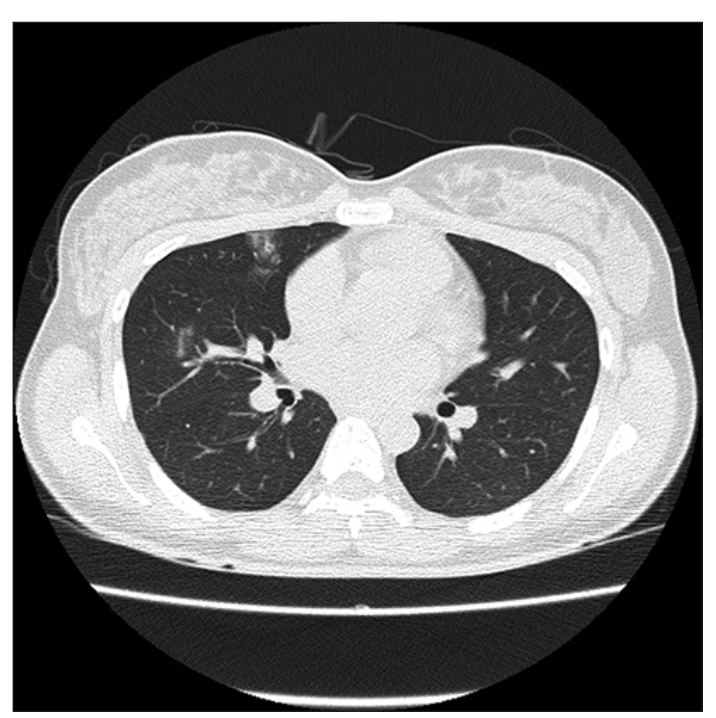

Fig. 1. Pre-cryotherapy chest computed tomography scan showing multiple endometrioses in the right and left upper lobes, December 2018.

tips $1.47 \mathrm{~mm}$ in diameter (Galil Medical, Yokneam, Israel). The patient was placed in the prone position, and the 2 cryoprobes were inserted into the centers of the endometrial lesions in the right lung under local anesthesia using 

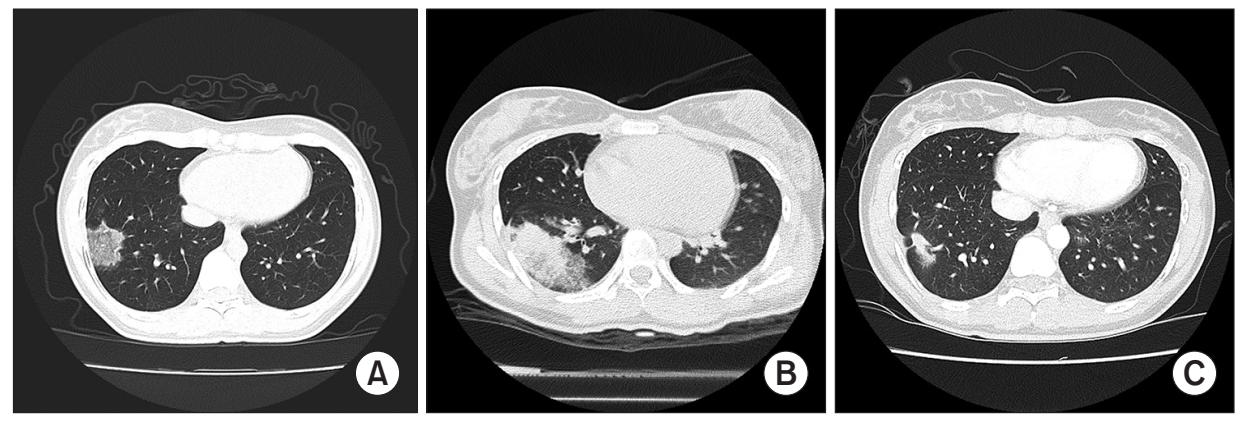

Fig. 2. (A) Pre-cryotherapy chest CT scan showing a large endometrial lesion on the right lower lobe, December 2018. (B) Post-cryotherapy chest CT scan showing a large iceball shadow on the right lower lobe 1 day after cryotherapy, January 2019. (C) Post-cryotherapy chest CT scan showing a decreased size of the endometrial lesion 3 months after cryotherapy, April 2019. CT, computed tomography.

$2.0 \%$ lidocaine. Throughout the procedure, the patient's blood pressure, echocardiogram, and oxygen saturation were monitored. Freezing and thawing were repeated 3 times each in accordance with the freeze-thaw protocol. The freezing process was conducted using argon gas and continued until the temperature of the tip of the cryoprobe was $-40^{\circ} \mathrm{C}$ (beginning at $-20^{\circ} \mathrm{C}$ ). This process took 3,7 , and 10 minutes for the first, second, and third repetitions, respectively. The thawing process was conducted using helium gas and continued until the temperature of the tip reached $20^{\circ} \mathrm{C}$; this took 3 minutes for the first repetition, 5 minutes for the second, and 2 minutes for the third.

The postoperative course was uneventful, and the patient was discharged 3 days after the cryoablation procedure. A follow-up chest CT scan was performed 1 day after the procedure and showed a large ice-ball shadow, despite the complete destruction of the endometrial lesions (Fig. 2B). Another follow-up chest CT scan was performed 3 months after the cryoablation procedure and showed a marked decrease in the size of the ice-ball shadow, suggesting complete eradication of the endometrial lesions (Fig. 2C). The patient will undergo regular follow-up at our outpatient clinic, and if symptoms of hemoptysis recur, she will be treated with another round of cryoablation.

The patient provided written informed consent for the publication of the clinical details and images associated with her case.

\section{Discussion}

Suspicion of pulmonary endometriosis in women is generally confirmed based on a clinical history of catamenial hemoptysis, active bleeding as observed via bronchoscopy, and evidence from CT scans. However, in most previously reported cases of pulmonary endometriosis, diagnosis was based primarily on the patient's history of periodic hemoptysis concurrent with menstruation. Based on these previous reports, ectopic endometriosis is not always confirmed histologically. The pathogenesis of endometriosis is attributed to lymphatic and venous dissemination of the endometrial tissue to distant sites with subsequent implantation and proliferation. CT findings associated with pulmonary endometriosis can include well-defined opacities, nodular lesions, thin-wall cavities, or bullous formations; however, most cases involving hemoptysis exhibit transient radiologic densities in the affected part of the lung. Pulmonary endometriosis is not correlated with the presence of pelvic endometriosis [1]. In the case of our patient, diagnosis was based on her clinical history and CT scan results. CT images taken during her menstrual period before cryoablation showed radiographic opacities in multiple areas.

Cryosurgery was first introduced in the mid-1850s and was further advanced throughout the 1960s with the introduction of the automatic apparatus [2]. Cryoablation is a percutaneous thermal treatment option that involves the use of argon and helium gases to decrease the temperature around the tip of the cryoprobe (the Thomson effect), freezing and thawing the tissue to destroy tumor cells [3]. In vivo and in vitro experiments have contributed to the understanding of the mechanisms of injury in various organs, such as the skin, liver, kidney, prostate, pancreas, and trachea/bronchus/lung [2]. Relatively non-invasive management techniques, including thermal ablation, radiofrequency, and cryoablation, may be considered in lung tumor patients with high surgical risk or inoperable metastatic lung cancer [4]. Cryoablation of lung tumors is usually carried out under local anesthesia and conscious sedation with chest CT guidance. The freezing-thawing process is performed 3 times, in accordance with the freeze-thaw protocol. Freezing is carried out 3 times, for several minutes per round, until the temperature of the tip of the cryoprobe reaches $-40^{\circ} \mathrm{C}$ (from $-20^{\circ} \mathrm{C}$ ). Thawing usually takes several minutes and is carried out until the temperature of the tip of the cryoprobe reaches $20^{\circ} \mathrm{C}$ [5].

Several mechanisms, including protein denaturation, cell 
destruction via osmotic shifts in extracellular and intracellular water-to-tissue ischemia, and microvascular thrombosis, are known to cause cellular injury as a result of cryoablation. As the temperature falls into the freezing range, ice crystals begin to form in the extracellular spaces, causing cell dehydration. As the temperature decreases further, ice crystals may form within the cells; this intracellular ice formation, which disrupts cell function, is a serious threat to cell viability. The disruption of organelles and cell membranes can lead to cell death. Furthermore, vascular stasis and thrombosis following cold injury are also well-known mechanisms of cell injury associated with cryoablation.

Common complications of cryoablation include cryoablation scars as well as acute phase reactions (such as alveolar hemorrhage, necrotic debris, inflammation, and edema) around the target tumor. As such, assessing the response in detail after cryoablation is difficult. Compared with the previous scan, all ablation zones should be significantly enlarged immediately after cryoablation. Following the acute phase reactions, all ablation zones should be decreased in size by the 30th and 180th days after cryoablation. Moreover, the shapes of the ablation zones after cryoablation can be classified into 5 patterns: consolidation/atelectasis, nodular, stripe, pleural thickening, and disappearance.

To date, to the best of our knowledge, cryoablation has not been used to treat pulmonary endometriosis; as such, we referenced previous cryoablation treatments used for benign lung tumors. Percutaneous cryoablation for lung tumors can be performed in a minimally invasive manner, with very low complication rates. The most common complication of cryoablation is pneumothorax. This complication occurs in approximately $12 \%$ to $62 \%$ of patients after cryoablation, and among them, approximately $12 \%$ require chest tube insertion. The occurrence of pneumothorax during or after cryoablation is related to the number or size ( $8 \mathrm{G}$ to $11 \mathrm{G}$ ) of the cryoprobes, and the rate of pneumothorax after cryoablation has been shown to decrease with the use of thin cryoprobes (17G). Another common complication is pleural effusion. Most pleural effusions are self-limiting and resolve with conservative management. The reported rates of hemoptysis after cryoablation range from $0 \%$ to $62 \%$, and in most cases, hemoptysis was selflimiting and was resolved with conservative management. Other known complications after cryoablation include cough, skin injuries, frostbite, empyema, arm paresis, temporary aphasia, phrenic nerve palsy, tumor implantation, and subcutaneous emphysema, to name a few [6].
Cryoablation is considered to be useful for the eradication of benign lung tumors; however, complete eradication of endometrial lesions with surgery may not be possible in cases of multiple variable-sized endometrioses in both lungs [6,7]. For curative cryoablation, the margin of the ice ball should extend 3 to $10 \mathrm{~mm}$ beyond the tumor margins [8]. Larger tumors, with a diameter of greater than $3.5 \mathrm{~cm}$, are not sufficiently covered by the ice ball. Another problem with cryoablation is that the ablated area of lung parenchyma around a lung tumor cannot be clearly differentiated from the margin of the ice ball due to the presence of equal water content.

However, in cases of pulmonary endometriosis, the actual endometrial lesions are usually less than $3.5 \mathrm{~cm}$ in diameter, despite being shown on the pre-cryoablation chest CT as having a diameter of greater than $5.0 \mathrm{~cm}$ due to hemorrhages around the endometrial lesion. In the case of our patient, the largest endometrial lesion (including hemorrhages) was smaller than $3.5 \mathrm{~cm}$, and we targeted the center of the lesion. Cryoablation can be performed repeatedly for recurrent pulmonary endometriosis. Therefore, it can be a feasible option for patients with multiple recurrent endometrioses who may be unfit for surgery.

\section{Conflict of interest}

No potential conflict of interest relevant to this article was reported.

\section{ORCID}

Chong Hoon Kim: https://orcid.org/0000-0001-7833-3882

Doo Yun Lee: https://orcid.org/0000-0003-3817-7066

Duk Hwan Moon: https://orcid.org/0000-0003-1388-2471

Sungsoo Lee: https://orcid.org/0000-0001-8998-9510

\section{References}

1. Huang H, Li C, Zarogoulidis P, et al. Endometriosis of the lung: report of a case and literature review. Eur J Med Res 2013;18:13.

2. Gage AA, Baust JM, Baust JG. Experimental cryosurgery investigations in vivo. Cryobiology 2009;59:229-43.

3. Inoue M, Nakatsuka S, Yashiro H, et al. Percutaneous cryoablation of lung tumors: feasibility and safety. J Vasc Interv Radiol 2012;23: 295-302.

4. Maiwand MO. The role of cryosurgery in palliation of tracheo-bronchial carcinoma. Eur J Cardiothorac Surg 1999;15:764-8.

5. Rodgers BM, Blake KD, Alexander JA. The effects of profound cryotherapy upon the pulmonary parenchyma. J Thorac Cardiovasc 
Surg 1982;83:784-9.

6. Wang H, Littrup PJ, Duan Y, Zhang Y, Feng H, Nie Z. Thoracic masses treated with percutaneous cryotherapy: initial experience with more than 200 procedures. Radiology 2005;235:289-98.

7. Moorjani N, Beeson JE, Evans JM, Maiwand MO. Cryosurgery for the treatment of benign tracheo-bronchial lesions. Interact Cardiovasc Thorac Surg 2004;3:547-50.

8. Gage AA, Guest K, Montes M, Caruana JA, Whalen DA Jr. Effect of varying freezing and thawing rates in experimental cryosurgery. Cryobiology 1985;22:175-82. 